\title{
Initial experience with a SiPM-based PET/CT scanner: influence of acquisition time on image quality
}

Ida Sonni ${ }^{1,3}$, Lucia Baratto ${ }^{1}$, Sonya Park ${ }^{1}$, Negin Hatami ${ }^{1}$, Shyam Srinivas ${ }^{1}$, Guido Davidzon', Sanjiv Sam Gambhir, ${ }^{1,2}$ and Andrei lagaru ${ }^{*^{*}}$ (i)

\footnotetext{
* Correspondence:

aiagaru@stanford.edu

${ }^{1}$ Division of Nuclear Medicine and Molecular Imaging, Department of Radiology, Stanford University, 300 Pasteur Drive, Stanford, CA 94305, USA

Full list of author information is available at the end of the article
}

\begin{abstract}
Background: A newly introduced PET/CT scanner (Discovery Meaningful Insights -DMI, GE Healthcare) includes the silicon photomultiplier (SiPM) with timeof-flight (TOF) technology first used in the GE SIGNA PET/MRI. In this study, we investigated the impact of various acquisition times on image quality using this SiPM-based PET/CT.

Methods: We reviewed data from 58 participants with cancer who were scanned using the DMI PET/CT scanner. The administered dosages ranged 295.3-429.9 MBq (mean \pm SD $356.3 \pm 37.4$ ) and imaging started at 71-142 min (mean \pm SD $101.41 \pm 17$. 52) after administration of the radiopharmaceutical. The patients' BMI ranged 19.79-46. 16 (mean \pm SD $26.55 \pm 5.53$ ). We retrospectively reconstructed the raw TOF data at 30, 60,90 , and $120 \mathrm{~s} /$ bed and at the standard image acquisition time per clinical protocol (180 or $210 \mathrm{~s} /$ bed depending on BMI). Each reconstruction was reviewed blindly by two nuclear medicine physicians and scored 1-5 (1-poor, 5-excellent quality). The liver signal-to-noise ratio (SNR) was used as a quantitative measure of image quality.

Results: The average scores \pm SD of the readers were $2.61 \pm 0.83,3.70 \pm 0.92,4.36 \pm 0.82$, $4.82 \pm 0.39$, and $4.91 \pm 0.91$ for the 30,60, 90, and $120 \mathrm{~s} /$ bed and at standard acquisition time, respectively. Inter-reader agreement on image quality assessment was good, with a weighted kappa of 0.80 (95\% Cl 0.72-0.81). In the evaluation of the effects of time per bed acquisition on semi-quantitative measurements, we found that the only time point significantly different from the standard time were 30 and $60 \mathrm{~s}$ (both with $P<0.001$ ). The effects of dose and BMI were not statistically significant ( $P=0.195$ and 0.098 , respectively). There was a significant positive effect of time on SNR $(P<0.001)$, as well as a significant negative effect of weight $(P<0.001)$.
\end{abstract}

Conclusions: Our results suggest that despite significant delays from injection to imaging (due to comparison with standard PET/CT) compared to standard clinical operations and even in a population with average BMI $>25$, images can be acquired as fast as $90 \mathrm{~s} /$ bed using the SiPM PET/CT and still result in very good image quality (average score $>4$ ).

Keywords: PET/CT, Acquisition time, Silicon photomultipliers, Detectors 


\section{Background}

Positron emission tomography (PET) is a functional imaging technique developed in the 1970s that is now an indispensable tool in the evaluation of biological functions at molecular level [1, 2]. PET plays a particularly crucial role in cancer imaging, for diagnostic, staging, and prognostic purposes [3]. After initial use as PET alone, the first hybrid PET/CT scanner was introduced and evaluated clinically in 2000 [4]. Hybrid PET/CT scanners benefit from the addition of structural information derived from CT and its important ability to provide maps of tissue attenuation for correction of the PET images, which have significantly improved the duration of the exam, patients' compliance, and ultimately diagnostic accuracy [5]. A substantial improvement in PET/CT is time-of-flight (TOF) capability. TOF allows for better localization of the annihilation event along the lines-of-response due to the use of fast detectors and ultimately improves the tomographic reconstruction and image quality [6]. More recently, simultaneous PET/MRI scanners have also been developed with the expectation to improve workflows and clinical usefulness in those fields where the properties of MRI are valuable [7-10]. Such combined and simultaneous scanners required remarkable modifications in PET technology over the years to move away from photomultiplier tubes.

Since their initial development to current times, most PET scanners were built with scintillation crystals coupled with PMTs. However, in recent years SiPM detectors emerged as a new technology foreseen as a promising alternative to PMTs [11]. These photodetectors offer several advantages including relative indifference to the magnetic fields of MR which made them attractive for the use in hybrid PET/MR systems, compact size, and potentially low production costs [11, 12]. Furthermore, SiPM are characterized by excellent intrinsic time resolution and high photon-detection efficiency, which made them the most favorable devices to be coupled with TOF reconstruction [12]. The differences between standard and SiPM PET detectors are significant. SiPMs having a small physical profile and a negligible electronic noise, offering high gain, and requiring low operating voltage offer several advantages over previously used technologies such as photomultiplier tubes (PMTs) and avalanche photodiodes (APDs) $[13,14]$. Among the potential advantages of SiPM-based PET/CT over standard $\mathrm{PET} / \mathrm{CT}$ are decreased required dosage of PET radiopharmaceuticals, higher sensitivity, and temporal resolution. Our group has previously reported the first experiences using SiPM-based detectors in the SIGNA PET/MRI $[15,16]$.

We installed in August 2016 the first SiPM-based clinical PET/CT (Discovery Meaningful Insights (DMI), GE Healthcare, Waukesha, WI, USA) that brings together SiPM and TOF [17]. Our hypothesis was that one can scan faster using the DMI without a compromise in image quality. Here, we report our initial results from the evaluation of the impact of various acquisition times on image quality using this SiPMbased PET/CT scanner.

\section{Methods}

\section{Patient population}

The study was approved by the Institutional Review Board (IRB) at Stanford University. Written informed consent was obtained from each participant before enrollment. Between September and December 2016, adult patients (18 years of age or older) 
referred to the Division of Nuclear Medicine and Molecular Imaging at Stanford University for a clinical oncological ${ }^{18}$ F-fluorodeoxyglucose $\left({ }^{18} \mathrm{~F}\right.$-FDG) PET/CT examination were selected and enrolled in our study. Patients unable to lie still for the duration of the exam and pregnant women were excluded.

\section{Study design and PET/CT protocol}

This is a retrospective analysis of data acquired prospectively in a study comparing the performance of standard PET/CT scanners (GE Discovery $600 \mathrm{PET} / \mathrm{CT}$ scanner and GE Discovery 690 PET/CT scanner) and the GE DMI SiPM-based PET/CT scanner. Patients underwent a single injection of ${ }^{18} \mathrm{~F}-\mathrm{FDG}$, followed by a dual-imaging PET

Table 1 Demographic and clinical data of all study participants

\begin{tabular}{|c|c|}
\hline Characteristics & Number of patients \\
\hline Total number of participants & 58 \\
\hline Male & 50 \\
\hline Female & 8 \\
\hline $\begin{array}{l}\text { Average age ( } \pm \text { standard deviation) } \\
\text { Age range }\end{array}$ & $\begin{array}{l}61.6 \text { years }( \pm 15) \\
27-94 \text { years }\end{array}$ \\
\hline \multicolumn{2}{|l|}{ Diagnosis } \\
\hline Lung cancer & 10 \\
\hline Non-Hodgkin lymphoma & 9 \\
\hline Head and neck cancer & 5 \\
\hline Hodgkin lymphoma & 5 \\
\hline Colorectal cancer & 4 \\
\hline Melanoma & 4 \\
\hline Breast cancer & 3 \\
\hline Testicular cancer & 2 \\
\hline Renal cell carcinoma & 1 \\
\hline Kidney cancer & 1 \\
\hline Myocardial sarcoidosis & 1 \\
\hline Bladder cancer & 1 \\
\hline Endometrial cancer & 1 \\
\hline Chronic lymphocytic leukemia & 1 \\
\hline Angiosarcoma & 1 \\
\hline Hepatocellular carcinoma & 1 \\
\hline Esophageal cancer & 1 \\
\hline Thyroid cancer & 1 \\
\hline Adrenocortical carcinoma & 1 \\
\hline Gastric cancer & 1 \\
\hline Pulmonary sarcoidosis & 1 \\
\hline Adenocarcinoma of unknown primary & 1 \\
\hline Lymphoadenopathy, splenomegaly & 1 \\
\hline IgA K multiple Myeloma & 1 \\
\hline \multicolumn{2}{|l|}{ Clinical indication } \\
\hline Initial treatment strategy & 11 \\
\hline Subsequent treatment strategy & 47 \\
\hline
\end{tabular}




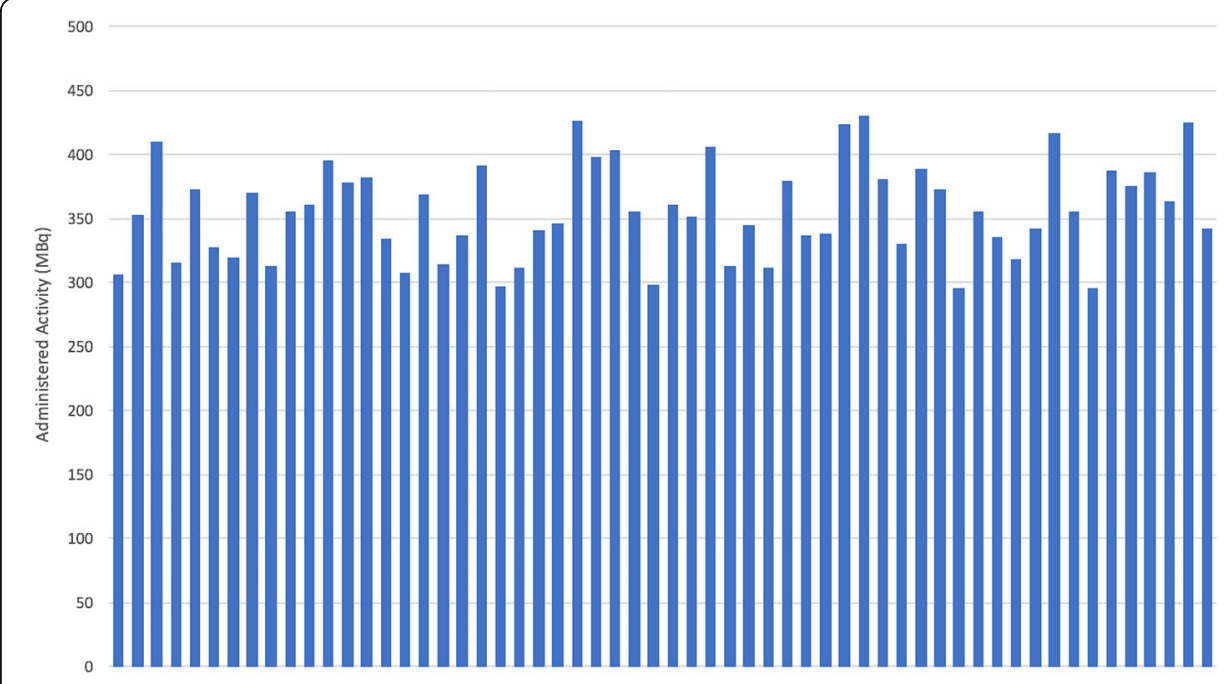

Fig. 1 Histogram of administered activity

protocol, including first the standard PET/CT, and immediately after the SiPM-based PET/CT. The DMI did not have FDA-clearance at the time the protocol was approved by the local ethics committee; therefore, standard PET/CT was always used first at standard time for oncological imaging.

Here, we only report on the PET datasets from the DMI PET/CT scanner. These were acquired in list mode. Subsequently, the PET list mode data was reprocessed to produce the following sets of sinograms: 30, 60, 90, and $120 \mathrm{~s}$ per bed (sec/bed). The reference standard acquisition time (reference time) varied between 180 and $210 \mathrm{~s}$ per bed, depending on the patients' BMI, as recommended by the manufacturer and to match the local clinical practice used for the D600 and D690 PET/CT scanners. All data sets were reconstructed using TOF with point spread function (PSF) and 3 iterations, 16 subsets, and a 5-mm post-filter, standard normalized $z$ axis filtering, in a $256 \times 256$ matrix (voxel dimensions of $2.73 \times 2.73 \times$ 2.79). A 17 -slice overlap was used on the DMI PET/CT scanner, which translates to $24 \%$ percentage of bed overlap. The average number of beds per patient, calculated dividing patient's height by axial field of view $(20 \mathrm{~cm})$ for a head-to-toe scan, was 9 , whereas for head-to-mid-thigh acquisitions, it was 6 . The CT protocol utilized a 40-mm detector coverage, with a slice thickness of $3.75 \mathrm{~mm}$, slice interval of $2.79 \mathrm{~mm}$ to match the PET slice thickness, and a pitch of 0.984 with a $0.5 \mathrm{~s}$ rotation time. The $\mathrm{kV}$ was set to 120 and the $\mathrm{mA}$ was set to modulate between 100 and 165 with a Noise Index of 25.00.

Table 2 Image quality assessment by the two readers. Rates in the rows are by reader 1 and rates in the columns are by reader 2 . Weighted kappa between readers was 0.73 ( $95 \% \mathrm{Cl} 0.66-0.80$ )

\begin{tabular}{lllllll}
\hline & 1 & 2 & 3 & 4 & 5 & Total (\%) \\
\hline 1 & 2 & 6 & 2 & 1 & 0 & $11(3.8 \%)$ \\
2 & 0 & 9 & 13 & 6 & 2 & $30(10.3 \%)$ \\
3 & 0 & 3 & 27 & 16 & 6 & $52(17.9 \%)$ \\
4 & 0 & 1 & 9 & 21 & 33 & $64(22.1 \%)$ \\
5 & 0 & 0 & 1 & 21 & 111 & $133(45.9 \%)$ \\
Total (\%) & $2(0.7 \%)$ & $19(6.6 \%)$ & $52(17.9 \%)$ & $65(22.4 \%)$ & $152(52.4 \%)$ & 290 \\
\hline
\end{tabular}


Table 3 Percentage of " 5 - excellent" ratings in relation to BMI

\begin{tabular}{lllllll}
\hline Time (s) & 30 & 60 & 90 & 120 & Reference time & Total \\
\hline BMI $\leq 25$ & $0 \%$ & $28 \%$ & $75 \%$ & $88 \%$ & $98 \%$ & $58 \%$ \\
& $0 / 60$ & $17 / 70$ & $45 / 60$ & $53 / 60$ & $59 / 60$ & $174 / 300$ \\
BMI > 25 & $0 \%$ & $7 \%$ & $36 \%$ & $75 \%$ & $82 \%$ & $40 \%$ \\
& $0 / 56$ & $4 / 56$ & $20 / 56$ & $42 / 56$ & $46 / 56$ & $112 / 280$ \\
\multirow{2}{*}{ Total } & $0 \%$ & $18 \%$ & $56 \%$ & $82 \%$ & $91 \%$ & $49 \%$ \\
& $0 / 116$ & $21 / 116$ & $65 / 116$ & $95 / 116$ & $105 / 116$ & $285 / 580$ \\
\hline
\end{tabular}

\section{Image analysis}

Images were reviewed and analyzed independently using Advantage Workstation (GE Healthcare). Two experienced nuclear medicine physicians (AI and IS, 11 and 6 years of experience interpreting PET scans, respectively) evaluated blindly all PET reconstructions for image quality. Image quality was rated using a 5-point Likert scale (5 excellent diagnostic image quality, 4 good, 3 acceptable, 2 suboptimal with limited additional clinical information, 1 non-diagnostic) in which a score of 3, 4, or 5 was considered to provide diagnostic value. One of the investigators (IS) identified one representative lesion per patient, and semi-quantitative measurements using maximum standardized uptake values $\left(\mathrm{SUV}_{\max }\right)$ were obtained for each reconstruction by drawing a spherical volume-of-interest (VOI) around the selected lesion. Additionally, the liver signal-to-noise ratio (SNR) was obtained for each of the time per bed reconstructions as a quantitative image quality parameter and surrogate of visual image quality. A 3-cm spherical VOI was placed within the right lobe of the liver. Liver SNR defined as the ratio of the voxel mean and the standard deviation of the activity in the VOI $[18,19]$.

\section{Statistical analysis}

Rating agreement for the image quality assessment between readers was evaluated using kappa statistic. Effects of time per bed on ratings and SNR were estimated by mixed-effects logistic regression with covariates of dose and BMI and random effect of patient. Effects of time per bed on SUV were estimated by mixed-effects GLM log-link regression with covariates of dose and BMI, and random effect of patient. All statistical analyses were done using Stata Release 14.2 (StataCorp LP, College Station, TX, USA). A significance level of 0.05 was used.

\section{Results}

A total of 58 cancer patients (50 men and 8 women; 27 - to 94-year-old, mean \pm SD $61.6 \pm 15$ years) were enrolled in the study; 41 patients had measurable lesions. Patients with all cancer types were included in the study to simulate the actual clinical

Table 4 Effects of time, BMl, and dose on ratings

\begin{tabular}{llllll}
\hline Variable & Odds ratio & Standard error & $Z$ & $P$ & $95 \%$ confidence interval \\
\hline Time/30 & 7.10 & 1.69 & 8.25 & 0.000 & $4.46-11.31$ \\
BMI (vs $\leq 25)$ & 3.38 & 2.59 & 1.59 & 0.112 & $0.75-15.14$ \\
BMI $\times$ time/30 & 0.38 & 0.10 & -3.85 & 0.000 & $0.23-0.62$ \\
Dose & 1.38 & 0.23 & 1.94 & 0.052 & $0.99-1.91$ \\
\hline
\end{tabular}




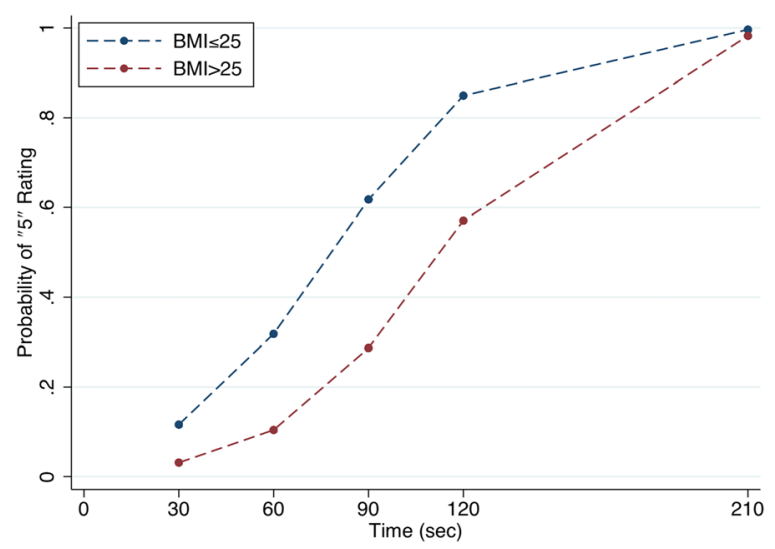

Fig. 2 Influence of acquisition time and BMI on image quality

experience. Relevant demographic and clinical data are showed in Table 1. DMI $\mathrm{PET} / \mathrm{CT}$ scans were acquired 101.41 $\pm 17.52 \mathrm{~min}$ (range 71-142 min) post-injection of $356.3 \pm 37.4 \mathrm{MBq}$ (range 295.3-429.9) of ${ }^{18} \mathrm{~F}$-FDG (Fig. 1). This time delay was due to standard PET/CT being acquired first. The wide range was mostly due to different duration of the initial scans, some of them lasting longer (head-to-toes) than others (head-to-mid-thighs) and, in some instances, due to accommodation of specific patient's needs. Patient's weight was $81.22 \pm 20.28 \mathrm{~kg}$ (range 57-143 kg) and body mass index (BMI) was $26.55 \pm 5.53$ (range 19.79-46.16; median 24.95). The recommended ${ }^{18}$ F-FDG dosage was $370-555 \mathrm{MBq}$ at the time of the DMI installation. Such variations in time from injection to imaging and in administered dosage have been reported at academic medical centers in the USA [20].

Image quality and semi-quantitative assessment

The average scores $\pm \mathrm{SD}$ of both readers were $2.61 \pm 0.83,3.70 \pm 0.92,4.36 \pm 0.82$, $4.82 \pm 0.39$, and $4.91 \pm 0.91$ for the $30,60,90,120 \mathrm{~s} / \mathrm{bed}$ and standard acquisition time, respectively.

Inter-reader agreement on image quality assessment was good, with a weighted kappa of 0.80 (95\% CI $0.72-0.81)$. Almost half of each reader's ratings were " 5 (Excellent Quality)"; their marginal distributions of ratings were significantly different (marginal homogeneity test $P<0.001$ ), and there was a significant tendency for rater 2's ratings to be higher than rater 1's when the two disagreed (symmetry test $P<0.001$ ) (Tables 2 and 3). Weighted kappa between readers was 0.73 (95\% CI $0.66-0.80)$. There was a significant overall effect of time on ratings $(P<0.001)$ as

Table 5 Means, SDs, and frequencies of SUV $\max$ measurements

\begin{tabular}{clllllllllllll}
\hline \multicolumn{1}{c}{ BMI $\leq 25$} & \multicolumn{10}{c}{ BMI $\geq 25$} \\
\hline Time (s) & 30 & 60 & 90 & 120 & $\begin{array}{l}\text { Reference } \\
\text { time }\end{array}$ & Total 30 & 60 & 90 & 120 & $\begin{array}{l}\text { Reference } \\
\text { time }\end{array}$ & Total \\
Mean & 10.67 & 10.68 & 10.60 & 10.49 & 10.43 & 10.57 & 13.06 & 12.36 & 12.04 & 11.92 & 11.92 & 12.26 \\
SD & 5.4 & 5.01 & 4.81 & 4.66 & 4.51 & 4.78 & 6.40 & 6.41 & 6.16 & 6.05 & 6.04 & 6.11 \\
Frequency & 20 & 20 & 20 & 20 & 20 & 100 & 21 & 21 & 21 & 21 & 21 & 105 \\
\hline
\end{tabular}




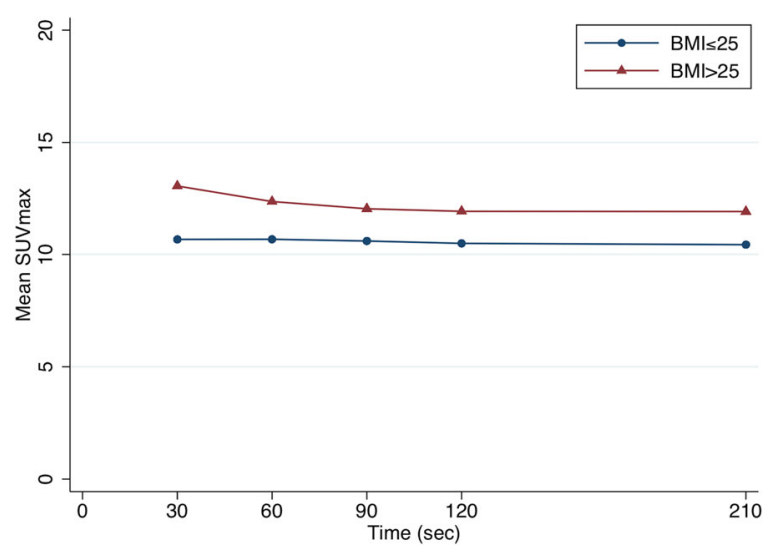

Fig. 3 Influence of acquisition time and BMI on SUV max

well as a significant time-by-BMI interaction $(P<0.001)$. There was no significant effect of dose $(P=0.052)$. These findings are summarized in Table 4 and Fig. 2.

In the evaluation of the effects of time per bed acquisition on semi-quantitative measurements we found that the only time-point significantly different from the standard time were 30 and $60 \mathrm{~s}$ (both with $P<0.001$ ). The effects of dose and BMI were not statistically significant $(P=0.195$ and 0.098 , respectively). These are shown in Table 5 and Fig. 3 . The percentage change of SUVmax relative to the reference time and to lesion volume is summarized in Table 6 and Fig. 4. The liver SNR obtained with each time per bed reconstruction are summarized in Tables 7 and 8 and Fig. 5a, b. There was a significant positive effect of time on SNR $(P<0.001)$, as well as a significant negative effect of weight $(P<0.001)$, and a possible positive effect of dose $(P=0.048)$. BMI and weight were highly correlated $(r=0.90)$; nevertheless, only weight had a statistically significant effect as predictor of the liver SNR, indicating that it shares more variance with SNR than BMI does.

Examples of various reconstructions in two different patients are shown in Figs. 6 and 7.

\section{Discussion}

Among the desired advantages of new PET systems are scanning time and dose reduction and increased image quality which would translate into better patient compliance, better and earlier lesion detection, and ultimately to better patient care and clinical management. Our study suggests that the SiPM technology and TOF reconstruction implemented in the DMI PET/CT scanner allow reduction of PET acquisition time to $90 \mathrm{~s} / \mathrm{bed}$, while still producing very good image quality. These may be even better under normal clinical circumstances, as our scans were acquired with a delay due to the standard PET/CT being done first. The high image quality ratings recorded for fast imaging opens other possibilities such as a combination of decreased dosage of the

Table 6 Percentage change of SUV $V_{\max }$ relative to reference time

\begin{tabular}{llll}
\hline Time $(\mathrm{s})$ & Mean & Standard error & 95\% confidence interval \\
\hline 30 & 6.01 & 2.24 & $1.48-10.53$ \\
60 & 2.08 & 1.33 & $-0.61-4.76$ \\
90 & 0.95 & 0.88 & $-0.81-2.73$ \\
120 & 0.14 & 0.63 & $-1.12-1.14$ \\
\hline
\end{tabular}




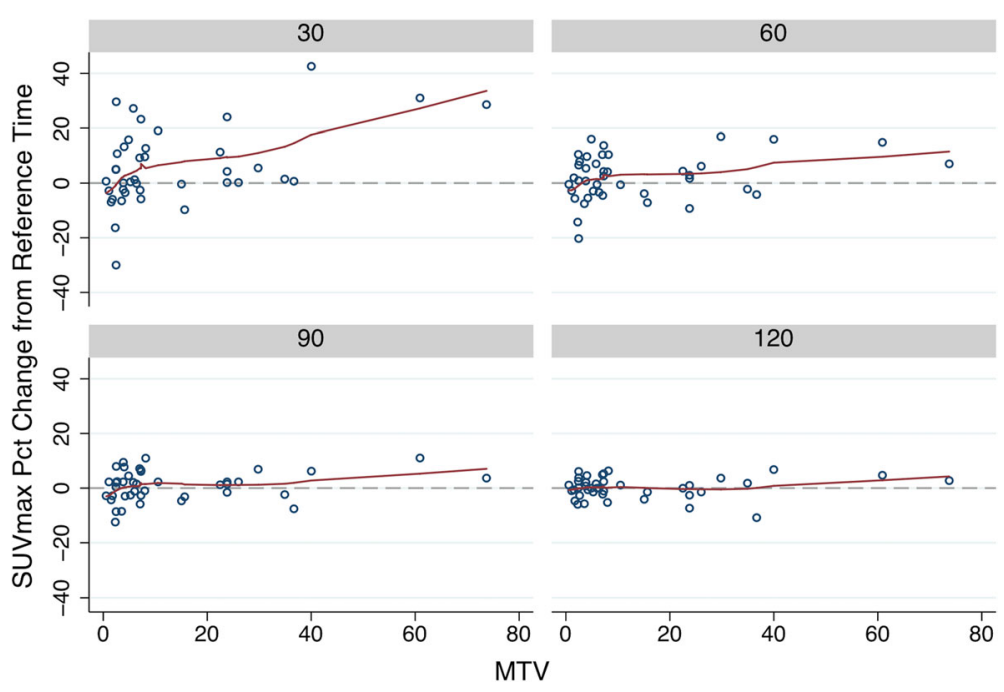

Fig. 4 Percentage change from standard time of SUVmax by lesion size. MTV mean tumor volume

administered radiopharmaceutical and fast imaging, which is now accepted as a compromise in our current use of DMI.

The recent introduction of SiPM detectors has attracted the interest of the scientific community due to the potential advantages of the new technology. These photodetectors are characterized by excellent intrinsic time resolution and high photon detection efficiency. The most attractive advantages derived from the use of SiPM-based PET/CT over standard PET/CT are a reduction in required administered dosage of PET radiopharmaceuticals, higher sensitivity and temporal resolution. Very little is available in the literature on the clinical use of the two technologies combined in PET/MR $[15,16]$ and PET/CT [21] scanners. A recently published study from our group using the DMI PET/CT system evaluated the performance of the novel scanner, in terms of spatial resolution, sensitivity, noise equivalent count rate (NECR), scatter fraction, count rate accuracy, and image quality with the National Electrical Manufacturers Association (NEMA) NU-2 2012 standards, finding excellent performance [21]. In comparison with other commercially available PET scanners from the same vendor, the DMI PET/CT performed better based on NEMA studies and showed the highest sensitivity, inferior only to the GE SIGNA PET/MR.

An important aspect of our data is that our population included many patients with a BMI $>25$. Our study indicates that as expected BMI negatively influences image quality. Due to the higher sensitivity, temporal and spatial resolution of the new technology implemented in the DMI PET/CT, a reduction in acquisition time is possible even in a population with high BMI, while maintaining image quality. It is true that, as expected,

Table 7 Effects of time, BMl, and dose on the liver SNR

\begin{tabular}{llllll}
\hline Variable & Odds ratio & Standard error & $Z$ & $P>|z|$ & $95 \%$ confidence interval \\
\hline Time/30 & 0.70 & 0.04 & 16.75 & 0.000 & $0.62-0.78$ \\
Dose & 0.29 & 0.15 & 1.98 & 0.048 & $0.00-0.57$ \\
Weight & -0.05 & 0.01 & -6.41 & 0.000 & $-0.06-0.03$ \\
(Intercept) & 4.88 & 1.49 & 3.29 & 0.001 & $1.97-7.80$ \\
\hline
\end{tabular}


Table 8 Means, SDs, and frequencies of liver SNR by BMI

\begin{tabular}{ccccclccccccc}
\hline \multicolumn{11}{c}{ BMI $\leq 25$} & \multicolumn{10}{c}{ BMI $\geq 25$} \\
\hline Time (sec) & 30 & 60 & 90 & 120 & Reference time & Total & 30 & 60 & 90 & 120 & Reference time & Total \\
Mean & 4.61 & 5.98 & 7.28 & 7.36 & 8.77 & 6.80 & 3.47 & 4.72 & 5.75 & 6.26 & 8.24 & 5.69 \\
SD & 1.66 & 1.17 & 1.77 & 2.27 & 2.41 & 2.35 & 1.47 & 1.38 & 1.59 & 2.16 & 2.33 & 2.41 \\
Frequency & 30 & 30 & 30 & 30 & 30 & 150 & 28 & 28 & 28 & 28 & 28 & 290 \\
\hline
\end{tabular}

image quality and liver SNR declined proportionally with acquisition time reduction, but our data showed that a scanning time of $90 \mathrm{~s} /$ bed still allows good image quality with an average score of $4.36 \pm 0.82$ in a 1 to 5 scale and liver SNR of $6.54 \pm 2.27$.

Two important aspects that we intend to explore in future studies are the effect of acquisition time reduction on lesion detectability and the possibility of dose reduction. No such analysis was performed in the current study since we chose to focus only on the impact of scanning time duration on image quality and semi-quantitative measurements. One limitation to our study is the delay between injection time and PET/CT
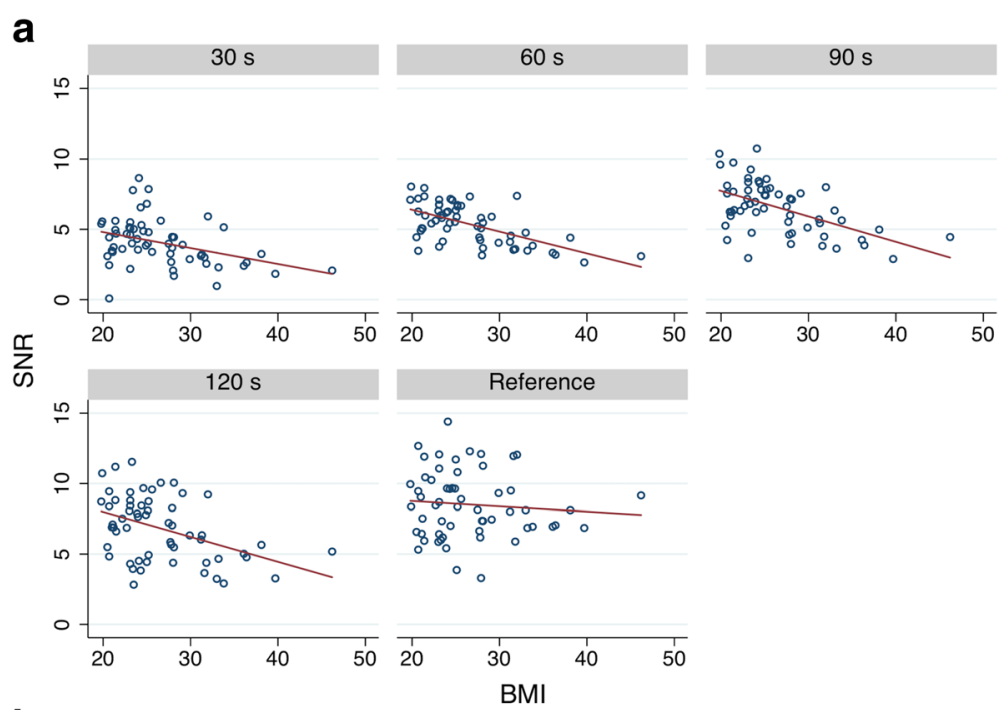

b
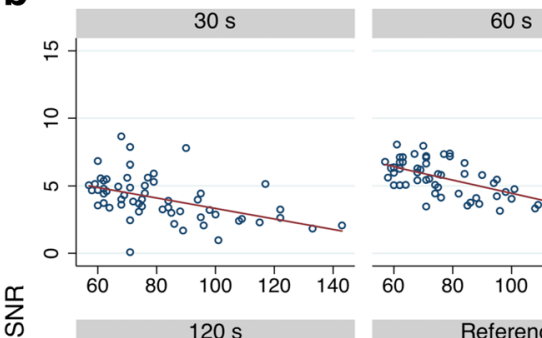

$90 \mathrm{~s}$

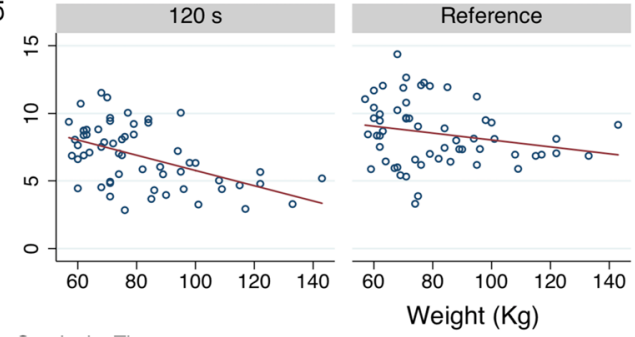

Fig. 5 Linear regression analysis correlating liver SNR with BMI (a) and body weight in kilogram (b) 


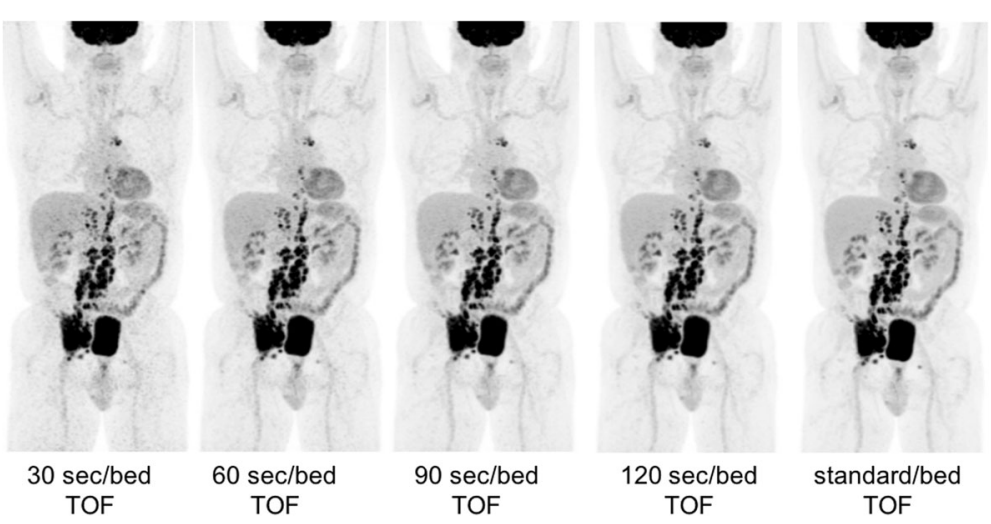

Fig. 6 Images acquired $71 \mathrm{~min}$ after injection of $423.3 \mathrm{MBq}$ of FDG and reconstructed at various times/bed (BMI 26.6). Reader 1's scores for each time per bed reconstruction were, respectively, 4, 5, 5, 5, and 5; reader 2's scores were $3,4,5,5$, and 5

acquisition, with an average time between the radiopharmaceutical injection and the beginning of the DMI PET/CT acquisition of $101.41 \pm 17.52$ min (range 71-142). As already mentioned, this delay was due to the acquisition of a standard of care PET/CT before the DMI PET/CT, which was acquired for research purposes. The delay in acquisition time might influence image quality and SUV measurements, but it is not possible to foresee what kind of impact it might have had on our results. This aspect will be subject of further investigations.

\section{Conclusions}

Despite significant delays from injection to imaging compared to standard clinical operations and even in a population with average $\mathrm{BMI}>25$, images can

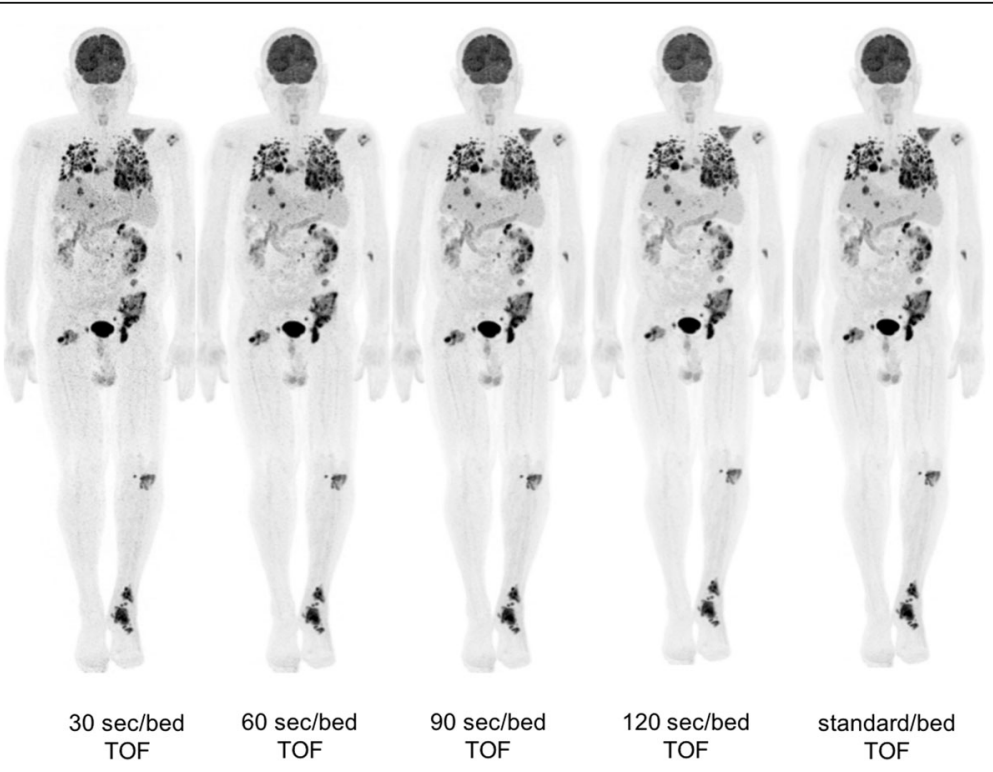

Fig. 7 Images acquired 116 min after injection of $330 \mathrm{MBq}$ of FDG and reconstructed at various times/bed (BMI: 21.2). Reader 1's scores for each time per bed reconstruction were respectively, 2, 4, 5, 5, and 5; reader 2's scores were 2, 3, 5, 5, and 5 
be acquired as fast as $90 \mathrm{~s} /$ bed using the SiPM-based DMI PET/CT and still result in very good image quality (average score $>4$ ). Fast PET/CT scanning may be significant for pediatric patient (decreasing anesthesia or sedation time) or for sick patients who cannot lay still for prolonged periods of time. In addition, increased patients' throughput may change workflows in busy clinical practices.

\section{Acknowledgements}

The authors would like to thank Jarrett Rosenberg, Ph.D., for his support to the statistical analysis.

\section{Funding}

This study was funded by GE Healthcare.

\section{Authors' contributions}

IS and Al took part in the study design, data collection and analysis, and manuscript preparation. LB contributed in the data collection, data analysis, and manuscript preparation. SP contributed in the data collection and data analysis. $\mathrm{NH}$ contributed in the data collection. GD and SS helped in the data analysis. SSG contributed in the study design. All authors read and approved the final manuscript.

Ethics approval and consent to participate

All procedures performed in studies involving human participants were in accordance with the ethical standards of the institutional and/or national research committee and with the 1964 Helsinki declaration and its later amendments or comparable ethical standards.

\section{Consent for publication}

Informed consent was obtained from all individual participants included in the study.

\section{Competing interests}

Guido Davidzon, Shyam Srinivas, and Andrei lagaru received institutional research grants from GE Healthcare. The remaining authors declare that they have no competing interests.

\section{Publisher's Note}

Springer Nature remains neutral with regard to jurisdictional claims in published maps and institutional affiliations.

\section{Author details}

${ }^{1}$ Division of Nuclear Medicine and Molecular Imaging, Department of Radiology, Stanford University, 300 Pasteur Drive, Stanford, CA 94305, USA. ${ }^{2}$ Molecular Imaging Program at Stanford, Department of Radiology, Stanford University, Stanford, CA, USA. ${ }^{3}$ Molecular Biophysics and Integrated Bioimaging, Lawrence Berkeley National Lab, Berkeley, CA, USA.

Received: 25 August 2017 Accepted: 21 February 2018

Published online: 18 April 2018

\section{References}

1. Ter-Pogossian MM, Phelps ME, Hoffman EJ, Mullani NA. A positron-emission transaxial tomograph for nuclear imaging (PETT). Radiology. 1975;114:89-98. https://doi.org/10.1148/114.1.89.

2. Phelps ME, Hoffman EJ, Mullani NA, Ter-Pogossian MM. Application of annihilation coincidence detection to transaxial reconstruction tomography. J Nucl Med. 1975;16:210-24.

3. Gambhir SS. Molecular imaging of cancer with positron emission tomography. Nat Rev Cancer. 2002;2:683-93. https://doi.org/10.1038/nrc882.

4. Beyer T, Townsend DW, Brun T, Kinahan PE, Charron M, Roddy R, et al. A combined PET/CT scanner for clinical oncology. J Nucl Med. 2000;41:1369-79.

5. Miles KA. PET-CT in oncology: making the most of CT. Cancer Imaging. 2008;8 Spec no A:S87-S93. https://doi.org/ 10.1102/1470-7330.2008.9015

6. Kadrmas DJ, Casey ME, Conti M, Jakoby BW, Lois C, Townsend DW. Impact of time-of-flight on PET tumor detection. J Nucl Med. 2009;50:1315-23. https://doi.org/10.2967/jnumed.109.063016.

7. Schlemmer HP, Pichler BJ, Schmand M, Burbar Z, Michel C, Ladebeck R, et al. Simultaneous MR/PET imaging of the human brain: feasibility study. Radiology. 2008;248:1028-35. https://doi.org/10.1148/radiol.2483071927.

8. Shao Y, Cherry SR, Farahani K, Meadors K, Siegel S, Silverman RW, et al. Simultaneous PET and MR imaging. Phys Med Biol. 1997:42:1965-70.

9. Barthel H, Schroeter ML, Hoffmann KT, Sabri O. PET/MR in dementia and other neurodegenerative diseases. Semin Nucl Med. 2015;45:224-33. https://doi.org/10.1053/j.semnuclmed.2014.12.003.

10. Fraum TJ, Fowler KJ, McConathy J. PET/MRI: emerging clinical applications in oncology. Acad Radiol. 2016; 23:220-36. https://doi.org/10.1016/j.acra.2015.09.008

11. Roncali E, Cherry SR. Application of silicon photomultipliers to positron emission tomography. Ann Biomed Eng. 2011;39:1358-77. https://doi.org/10.1007/s10439-011-0266-9. 
12. Ullah MN, Pratiwi E, Cheon J, Choi H, Yeom JY. Instrumentation for time-of-flight positron emission tomography. Nucl Med Mol Imaging. 2016;50:112-22. https://doi.org/10.1007/s13139-016-0401-5.

13. Grant AM, Lee BJ, Chang CM, Levin CS. Simultaneous PET/MR imaging with a radio frequency-penetrable PET insert. Med Phys. 2017:44:112-20. https://doi.org/10.1002/mp.12031.

14. Seifert S, van der Lei G, van Dam HT, Schaart DR. First characterization of a digital SiPM based time-offlight PET detector with 1 mm spatial resolution. Phys Med Biol. 2013;58:3061-74. https://doi.org/10.1088/ 0031-9155/58/9/3061.

15. lagaru A, Mittra E, Minamimoto R, Jamali M, Levin C, Quon A, et al. Simultaneous whole-body time-of-flight 18FFDG PET/MRI: a pilot study comparing SUVmax with PET/CT and assessment of MR image quality. Clin Nucl Med. 2015:40:1-8. https://doi.org/10.1097/rlu.0000000000000611.

16. Minamimoto R, Levin C, Jamali M, Holley D, Barkhodari A, Zaharchuk G, et al. Improvements in PET image quality in time of flight (TOF) simultaneous PET/MRI. Mol Imaging Biol. 2016;18:776-81. https://doi.org/10. 1007/s11307-016-0939-8

17. Baratto L, Park SY, Hatami N, Davidzon G, Srinivas S, Gambhir SS, et al. (18) F-FDG silicon photomultiplier PET/CT: a pilot study comparing semi-quantitative measurements with standard PET/CT. PLoS One. 2017;12:e0178936. https://doi.org/10.1371/journal.pone.0178936.

18. McDermott GM, Chowdhury FU, Scarsbrook AF. Evaluation of noise equivalent count parameters as indicators of adult whole-body FDG-PET image quality. Ann Nucl Med. 2013;27:855-61. https://doi.org/10.1007/s12149-0130760-2.

19. Fukukita H, Senda M, Terauchi T, Suzuki K, Daisaki H, Matsumoto K, et al. Japanese guideline for the oncology FDG-PET/CT data acquisition protocol: synopsis of version 1.0. Ann Nucl Med. 2010;24:325-34. https://doi.org/10. 1007/s12149-010-0377-7.

20. Graham MM, Badawi RD, Wahl RL. Variations in PET/CT methodology for oncologic imaging at U.S. academic medical centers: an imaging response assessment team survey. J Nucl Med. 2011;52:311-7. https://doi.org/10. 2967/jnumed.109.074104

21. Hsu DF, Ilan E, Peterson WT, Uribe J, Lubberink M, Levin CS. Studies of a next generation silicon-photomultiplierbased time-of-flight PET/CT system. J Nucl Med. 2017; https://doi.org/10.2967/jnumed.117.189514.

Submit your manuscript to a SpringerOpen ${ }^{\circ}$ journal and benefit from:

- Convenient online submission

- Rigorous peer review

- Open access: articles freely available online

High visibility within the field

- Retaining the copyright to your article

Submit your next manuscript at $>$ springeropen.com 